PROCEEDINGS OF THE

AMERICAN MATHEMATICAL SOCIETY

Volume 134, Number 2, Pages 355-364

S 0002-9939(05)08362-0

Article electronically published on September 21, 2005

\title{
THE VON NEUMANN-JORDAN CONSTANT, WEAK ORTHOGONALITY AND NORMAL STRUCTURE IN BANACH SPACES
}

\author{
ANTONIO JIMÉNEZ-MELADO, ENRIQUE LLORENS-FUSTER, AND SATIT SAEJUNG
}

(Communicated by Jonathan M. Borwein)

\begin{abstract}
We give some sufficient conditions for normal structure in terms of the von Neumann-Jordan constant, the James constant and the weak orthogonality coefficient introduced by B. Sims. In the rest of the paper, the von Neumann-Jordan constant and the James constant for the Bynum space $\ell_{2, \infty}$ are computed, and are used to show that our results are sharp.
\end{abstract}

\section{INTRODUCTION}

If $C$ is a subset of a Banach space $X$, a mapping $T: C \rightarrow X$ is called nonexpansive if $\|T(x)-T(y)\| \leq\|x-y\|$ for all $x, y \in C$. We say that $X$ has the fixed point property (FPP) if every nonexpansive self-mapping $T: C \rightarrow C$ of each nonempty, closed, bounded and convex subset $C$ of $X$ has a fixed point. The weak fixed point property (WFPP) is defined similarly by replacing closed and bounded by weakly compact. Obviously, for a reflexive Banach space (FPP) and (WFPP) are the same.

In the last forty years the question of whether a Banach space $X$ has, or has not, the (WFPP) has been intensively studied and although the list of sufficient conditions for (WFPP) (in most cases of geometrical type) is still increasing, perhaps the most celebrated result in this direction is the one given by W.A. Kirk [Ki], which states that those Banach spaces with weak normal structure have the (WFPP). Recall that the Banach space $X$ has weak normal structure ((WNS) for short) if every nonempty convex and weakly compact subset $C$ of $X$, with $\operatorname{diam}(C)>0$, contains a nondiametral point, that is, there exists $x_{0} \in C$ such that $\sup \left\{\left\|x-x_{0}\right\|: x \in C\right\}<$ $\operatorname{diam}(C)$. If we replace weakly compact by bounded and closed, we obtain the definition of normal structure (NS), which for reflexive spaces equals (WNS). For more information on this topic we refer the interested reader to [G-K], Kh Ki] and Ki S, for instance.

In a recent paper, M. Kato, L. Maligranda and Y. Takahashi KMT gave a sufficient condition for (NS) in terms of the von Neumann-Jordan constant $C_{\mathrm{NJ}}(X)$, which was defined in 1937 by J.A. Clarkson as

$$
C_{\mathrm{NJ}}(X)=\sup \left\{\frac{\|x+y\|^{2}+\|x-y\|^{2}}{2\left(\|x\|^{2}+\|y\|^{2}\right)}: x, y \in X \text { and }\|x\|+\|y\| \neq 0\right\} .
$$

Received by the editors January 23, 2004.

2000 Mathematics Subject Classification. Primary 46B20.

Key words and phrases. von Neumann-Jordan constant, James constant, normal structure.

(C)2005 American Mathematical Society 
Although some properties of $X$ such as uniform nonsquareness, superreflexivity, type, and cotype can be described in terms of the constant $C_{\mathrm{NJ}}(X)$ (see $[\mathrm{KT}$, KMT and the references therein), fixed point theorems in terms of this constant are uncommon in the literature. The only exception is, perhaps, Corollary 3 in KMT where the authors showed that if $C_{\mathrm{NJ}}(X)<5 / 4$, then $X$ has normal structure. (Even more, they proved that $X$ and $X^{*}$ have uniform normal structure.) This result has been recently improved by S. Dhompongsa, P. Piraisangjun and S. Saejung in DPS, where among other things, the authors show that $X$ has normal structure whenever $C_{\mathrm{NJ}}(X)<\frac{3+\sqrt{5}}{4}$.

Another coefficient which was used to give sufficient conditions for normal structure is the James constant $J(X)$ defined as

$$
\begin{aligned}
J(X) & =\sup \{\min (\|x+y\|,\|x-y\|):\|x\|=1,\|x\|=1\} \\
& =\sup \{\min (\|x+y\|,\|x-y\|):\|x\| \leq 1,\|x\| \leq 1\} .
\end{aligned}
$$

J. Gao and K.S. Lau proved in GL2 that a Banach space $X$ has normal structure if $J(X)<\frac{3}{2}$, and again this result has been recently improved by S. Dhompongsa, A. Kaewkhao and S. Tasena, who proved in DKT that the constant $\frac{3}{2}$ can be replaced by $\frac{1+\sqrt{5}}{2}$.

Let us mention another sufficient condition for normal structure given by B. Sims $[S$ ] in terms of a coefficient of weak orthogonality $\omega(X)$. As in [JL, we prefer to use its inverse, $\mu(X)$, which is defined as the infimum of the set of real numbers $r>0$ such that

$$
\limsup _{n}\left\|x+x_{n}\right\| \leq r \limsup _{n}\left\|x-x_{n}\right\|
$$

for all $x \in X$ and for all weakly null sequences $\left(x_{n}\right)$ in $X$.

B. Sims proved in $[S]$ that a sufficient condition for normal structure is the following: there exists $\varepsilon \in(0,2)$ such that

$$
\frac{1}{\mu(X)}>\max \left\{\frac{\varepsilon}{2}, 1-\delta_{X}(\varepsilon)\right\}
$$

where $\delta_{X}:[0,2] \rightarrow[0,1]$ is the usual modulus of convexity of $X$ defined as

$$
\delta_{X}(\varepsilon)=\inf \left\{1-\frac{1}{2}\|x+y\|: x, y \in B_{X},\|x-y\| \geq \varepsilon\right\} .
$$

In Section 2 we give two sufficient conditions for normal structure in terms of $C_{\mathrm{NJ}}(X), J(X)$ and $\mu(X)$, and in Section 3 we give the exact value of $C_{\mathrm{NJ}}(X)$ and $J(X)$ when $X$ is the Bynum space $\ell_{2, \infty}$. This solves a question posed in KMT] and also shows that our results are sharp.

\section{TWO SUFFICIENT CONDITIONS FOR NORMAL STRUCTURE}

As stated in the introduction, in this section we give two sufficient conditions for normal structure, and in the proof we shall use the following result (see, for instance, $\mathrm{KMT}$ ): if $C_{\mathrm{NJ}}(X)<2$ or $J(X)<2$, then $X$ is reflexive.

Theorem 1. If $X$ is a Banach space with $C_{\mathrm{NJ}}(X)<1+\frac{1}{\mu(X)^{2}}$, then $X$ has normal structure.

Proof. Observe that $C_{\mathrm{NJ}}(X)<2$ since $\mu(X) \geq 1$. Thus, $X$ is reflexive, and then normal structure equals weak normal structure. 
Suppose, looking for a contradiction, that $X$ lacks weak normal structure. Then, it is well known (cf. $[\mathrm{G}-\mathrm{K}]$ or $[\mathrm{P}]$ ) that there exists a bounded sequence $\left(x_{n}\right)$ in $X$ such that the following statements hold.

(i) $\left(x_{n}\right)$ is weakly convergent to $0_{X}$.

(ii) $\operatorname{diam}\left(\left\{x_{n}: n=1,2, \ldots\right\}\right)=1$.

(iii) For all $x \in \operatorname{clco}\left(\left\{x_{n}: n=1,2, \ldots\right\}\right)$ we have

$$
\lim _{n \rightarrow \infty}\left\|x-x_{n}\right\|=\operatorname{diam}\left(\left\{x_{n}: n=1,2, \ldots\right\}\right)=1 .
$$

Fix $\varepsilon>0$ as small as needed. Then, using the above properties of $\left(x_{n}\right)$ and the definition of $\mu(X)$ (for short: $\mu=\mu(X)$ ), we obtain two positive integers $m$ and $n$, with $m$ greater than $n$, such that

1) $\left\|x_{n}\right\| \geq 1-\varepsilon$.

2) $\left\|x_{m}-x_{n}\right\| \leq 1$.

3) $\left\|x_{m}+x_{n}\right\| \leq \mu+\varepsilon$.

4) $\left\|x_{m}-\frac{\mu^{2}-1}{\mu^{2}+1} x_{n}\right\| \geq 1-\varepsilon$.

5) $\left\|\left(\mu^{2}-1\right) x_{m}-\left(\mu^{2}+1\right) x_{n}\right\| \geq\left(\mu^{2}+1\right)\left\|x_{n}\right\|-\varepsilon$.

Next, put $x=\mu^{2}\left(x_{m}-x_{n}\right)$ and $y=x_{m}+x_{n}$ and use the previous estimates to obtain that $\|x\| \leq \mu^{2},\|y\| \leq \mu+\varepsilon$, and also that

$$
\begin{aligned}
\|x+y\| & =\left\|\left(\mu^{2}+1\right) x_{m}-\left(\mu^{2}-1\right) x_{n}\right\| \\
& =\left(1+\mu^{2}\right)\left\|x_{m}-\frac{\mu^{2}-1}{\mu^{2}+1} x_{n}\right\| \\
& \geq\left(1+\mu^{2}\right)(1-\varepsilon), \\
\|x-y\| & =\left\|\left(\mu^{2}-1\right) x_{m}-\left(\mu^{2}+1\right) x_{n}\right\| \\
& \geq\left(\mu^{2}+1\right)\left\|x_{n}\right\|-\varepsilon \\
& \geq\left(\mu^{2}+1\right)(1-\varepsilon)-\varepsilon .
\end{aligned}
$$

Then, by definition of $C_{\mathrm{NJ}}(X)$,

$$
\begin{aligned}
C_{\mathrm{NJ}}(X) & \geq \frac{\|x+y\|^{2}+\|x-y\|^{2}}{2\left(\|x\|^{2}+\|y\|^{2}\right)} \\
& \geq \frac{\left(1+\mu^{2}\right)^{2}(1-\varepsilon)^{2}+\left[\left(1+\mu^{2}\right)(1-\varepsilon)-\varepsilon\right]^{2}}{2 \mu^{4}+2(\mu+\varepsilon)^{2}} .
\end{aligned}
$$

Finally, letting $\varepsilon \rightarrow 0^{+}$, we obtain that

$$
\begin{aligned}
C_{\mathrm{NJ}}(X) & \geq \frac{\left(1+\mu^{2}\right)^{2}+\left(1+\mu^{2}\right)^{2}}{2 \mu^{4}+2 \mu^{2}} \\
& =1+\frac{1}{\mu^{2}}
\end{aligned}
$$

which contradicts the hypothesis.

Theorem 2. If $X$ is a Banach space with $J(X)<1+\frac{1}{\mu(X)}$, then $X$ has normal structure.

Proof. As in the previous theorem, our hypothesis implies that $X$ is reflexive because $J(X)<2$, so we just need to prove that $X$ has weak normal structure. We do it again by contradiction: suppose that $X$ lacks weak normal structure. Then, 
obtain a sequence $\left(x_{n}\right)$ satisfying the conditions i), ii) and iii) listed in Theorem 1. Now, fix $\varepsilon>0$ sufficiently small and obtain two positive integers $n, m$, with $m>n$, such that (for short: $\mu=\mu(X)$ )

(1) $\left\|x_{n}\right\| \geq 1-\varepsilon$.

(2) $\left\|x_{m}-x_{n}\right\| \leq 1$.

(3) $\left\|x_{m}+x_{n}\right\| \leq \mu+\varepsilon$.

(4) $\left\|\left(1-\frac{1}{\mu+\varepsilon}\right) x_{m}-\left(1+\frac{1}{\mu+\varepsilon}\right) x_{n}\right\| \geq\left(1+\frac{1}{\mu+\varepsilon}\right)\left\|x_{n}\right\|-\varepsilon$.

(5) $\left\|\left(1+\frac{1}{\mu+\varepsilon}\right) x_{m}-\left(1-\frac{1}{\mu+\varepsilon}\right) x_{n}\right\| \geq\left(1+\frac{1}{\mu+\varepsilon}\right)(1-\varepsilon)$.

Next, put $x=x_{m}-x_{n}$ and $y=(\mu+\varepsilon)^{-1}\left(x_{m}+x_{n}\right)$ and use the previous estimates to obtain that $\|x\| \leq 1,\|y\| \leq 1$, and also that

$$
\begin{aligned}
\|x+y\| & =\left\|\left(1+\frac{1}{\mu+\varepsilon}\right) x_{m}-\left(1-\frac{1}{\mu+\varepsilon}\right) x_{n}\right\| \\
& \geq\left(1+\frac{1}{\mu+\varepsilon}\right)(1-\varepsilon), \\
\|x-y\| & =\left\|\left(1-\frac{1}{\mu+\varepsilon}\right) x_{m}-\left(1+\frac{1}{\mu+\varepsilon}\right) x_{n}\right\| \\
& \geq\left(1+\frac{1}{\mu+\varepsilon}\right)\left(\left\|x_{n}\right\|-\varepsilon\right) \\
& \geq\left(1+\frac{1}{\mu+\varepsilon}\right)(1-\varepsilon)-\varepsilon .
\end{aligned}
$$

Then, by definition of $J(X)$,

$$
J(X) \geq \min \{\|x+y\|,\|x-y\|\} \geq\left(1+\frac{1}{\mu+\varepsilon}\right)(1-\varepsilon)-\varepsilon,
$$

and letting $\varepsilon \rightarrow 0^{+}$we obtain that $J(X) \geq 1+\frac{1}{\mu}$, which is a contradiction.

Remark 1. S. Dhompongsa, P. Piraisangjun and S. Saejung proved in DPS] that a Banach space $X$ has normal structure whenever $C_{\mathrm{NJ}}(X)<\frac{3+\sqrt{5}}{4}$. If we compare this result with Theorem 1, we observe that for those spaces with $\mu(X)$ close to 1 the result in DPS] does not apply but our Theorem 1 still gives information on the normal structure of $X$. The same can be said about Theorem 2 and the result given by S. Dhompongsa, A. Kaewkhao and S. Tasena, who proved in DKT] that $X$ has normal structure if $J(X)<\frac{1+\sqrt{5}}{2}$.

Remark 2. Theorem 2 recovers a result by B. Sims $[\underline{S}$. In this paper the author introduced the so-called property $(\mathrm{K})$ and proved that any Banach space with property $(\mathrm{K})$ has normal structure. Recall that a Banach space has property $(\mathrm{K})$ if there exists a real number $k$, with $0 \leq k<1$, such that

$$
\left.\begin{array}{l}
x_{n} \rightarrow 0 \\
\left\|x_{n}\right\| \rightarrow 1 \\
\liminf \left\|x_{n}-x\right\| \leq 1
\end{array}\right\} \Longrightarrow\|x\| \leq k \text {. }
$$


Moreover, Sims showed that the Banach space $X$ has property $(\mathrm{K})$ whenever

$$
\frac{1}{\mu(X)}>\max \left\{\frac{1}{2} \tau, 1-\delta_{X}(\tau)\right\}
$$

for some $\tau \in(0,2)$.

Observe that for any Banach space $X$ we have that

$$
\delta_{X}(\varepsilon) \leq 1-\sqrt{1-\frac{\varepsilon^{2}}{4}}
$$

$(\varepsilon \in[0,2])$, and then for every $\tau \in[0,2]$,

$$
\max \left\{\frac{1}{2} \tau, 1-\delta_{X}(\tau)\right\} \geq \max \left\{\frac{1}{2} \tau, \sqrt{1-\frac{\tau^{2}}{4}}\right\} \geq \frac{\sqrt{2}}{2} .
$$

Thus, if $\mu(X) \geq \sqrt{2}$, that is, if $\frac{1}{\mu(X)} \leq \frac{\sqrt{2}}{2}$, the above Sims' sufficient condition for property $(\mathrm{K})$ (and hence for WNS) cannot be fulfilled.

To see that the result of B. Sims is contained in Theorem 2 we need to prove that property $(*)$ implies that $J(X)<1+\frac{1}{\mu(X)}$. In fact we shall prove a stronger result (observe that $\frac{2}{\mu(X)} \leq 1+\frac{1}{\mu(X)}$ because $\mu(X) \geq 1$ ).

Claim. Condition $(*)$ is equivalent to $J(X)<\frac{2}{\mu(X)}$.

Indeed, if $J(X)<\frac{2}{\mu(X)}$, then for any real number $\tau$ with $J(X)<\tau<\frac{2}{\mu(X)}$ we have that

$$
\max \left\{\frac{1}{2} \tau, 1-\delta_{X}(\tau)\right\}=\frac{1}{2} \tau<\frac{1}{\mu(X)}
$$

because $J(X)=\sup \left\{\varepsilon \in(0,2): \frac{\varepsilon}{2}<1-\delta_{X}(\varepsilon)\right\}$ (see GL1] for instance) and $\tau>J(X)$.

For the converse suppose that

$$
\frac{1}{\mu(X)}>\max \left\{\frac{1}{2} \tau, 1-\delta_{X}(\tau)\right\}
$$

for some $\tau \in(0,2)$.

Consider two vectors $x, y$ in the closed unit ball of $X$ and let us see that

$$
\min \{\|x+y\|,\|x-y\|\}<\max \left\{\tau, 2\left(1-\delta_{X}(\tau)\right)\right\} .
$$

The argument is easy: if $\|x-y\|<\tau$ we have finished; otherwise, the definition of $\delta_{X}(\cdot)$ gives that

$$
\delta_{X}(\tau) \leq 1-\left\|\frac{1}{2}(x+y)\right\|,
$$

that is, $\|x+y\| \leq 2\left(1-\delta_{X}(\tau)\right)$. Now, use the relation $(*)$ to obtain that $J(X)<\frac{2}{\mu(X)}$.

In [DPS], [DKT] and [KMT] the authors give conditions which guarantee that both $X$ and $X^{*}$ have normal structure. We mention that under the hypothesis of our Theorem 1 we can also conclude the normal structure of $X^{*}$. To achieve this conclusion it suffices to bear in mind that $C_{\mathrm{NJ}}(X)=C_{\mathrm{NJ}}\left(X^{*}\right)$ and that $\mu(X)=$ $\mu\left(X^{*}\right)$ when $X$ is reflexive. The first fact is a well-known result and the second is proved in the following theorem.

Theorem 3. If a Banach space $X$ is reflexive, then $\mu(X)=\mu\left(X^{*}\right)$. 
Proof. Let $\left(x_{n}\right) \subset X$ be a weakly null sequence and $x \in X$. Take $f_{n} \in S_{X^{*}}$ so that $f_{n}\left(x_{n}-x\right)=\left\|x_{n}-x\right\|$. We choose subsequences $\left(x_{n_{k}}\right)$ of $\left(x_{n}\right)$ and $\left(f_{n_{k}}\right)$ of $\left(f_{n}\right)$ such that $\lim _{k}\left\|x_{n_{k}}-x\right\|=\lim \sup _{n}\left\|x_{n}-x\right\|$ and $f_{n_{k}} \stackrel{w}{\rightarrow} f$ for some $f \in X^{*}$. The last convergence follows from the reflexivity of $X$. Then

$$
\begin{aligned}
\underset{n}{\limsup \left\|x_{n}-x\right\|} & =\lim _{k}\left\|x_{n_{k}}-x\right\| \\
& =\lim _{k} f_{n_{k}}\left(x_{n_{k}}-x\right) \\
& =\lim _{k}\left(\left(f_{n_{k}}-f\right)-f\right)\left(x_{n_{k}}+x\right) \\
& \leq \limsup _{k}\left\|\left(f_{n_{k}}-f\right)-f\right\|\left\|x_{n_{k}}+x\right\| \\
& \leq \mu\left(X^{*}\right) \limsup _{k}\left\|f_{n_{k}}\right\| \limsup _{k}\left\|x_{n_{k}}+x\right\| \\
& =\mu\left(X^{*}\right) \limsup _{k}\left\|x_{n_{k}}+x\right\|,
\end{aligned}
$$

and hence $\mu(X) \leq \mu\left(X^{*}\right)$. By the reflexivity of $X$ again, we have the assertion.

Remark 3 . The reflexivity cannot be dropped. For example, it can be shown that $\mu\left(l_{1}\right)=1$ while $\mu\left(l_{\infty}\right)>1$.

Corollary 1. If $C_{\mathrm{NJ}}(X)<1+\frac{1}{\mu(X)^{2}}$, then $X$ and $X^{*}$ have normal structure.

Remark 4. The above argument cannot be emulated in Theorem 2 because in general $J(X) \neq J\left(X^{*}\right)$ (see [KMT] $)$.

\section{The Bynum space $\ell_{2, \infty}$}

W.L. Bynum [B] showed that a Banach space $X$ has normal structure whenever the Banach-Mazur distance from $X$ to $\ell_{p}, 1<p<\infty$, satisfies $d\left(X, \ell_{p}\right)<2^{\frac{1}{p}}$, but it still has the (FPP) if $d\left(X, \ell_{p}\right) \leq 2^{\frac{1}{p}}$. A sort of "limiting case" of Bynum's result is the space $\ell_{p, \infty}$, which is $\ell_{p}$ renormed according to $\|x\|_{p, \infty}=\max \left\{\left\|x^{+}\right\|,\left\|x^{-}\right\|\right\}$, where $x^{+}$and $x^{-}$are the positive and the negative part of $x$, respectively, defined as $x^{+}(i)=\max \{x(i), 0\}$ and $x^{-}=x^{+}-x$. In [KMT the authors posed the question of finding the exact value of $C_{\mathrm{NJ}}\left(\ell_{2, \infty}\right)$ and $J\left(\ell_{2, \infty}\right)$. It was noted there that $C_{\mathrm{NJ}}\left(\ell_{2, \infty}\right) \geq \frac{3}{2}$ and $J\left(\ell_{2, \infty}\right) \geq 1+\frac{1}{\sqrt{2}}$. To obtain the first inequality just consider the vectors $x=(1,1,0,0, \ldots)$ and $y=(2,-2,0,0, \ldots)$ and use the definition of the norm $\|\cdot\|_{2, \infty}$. For the second inequality consider the vectors $x=\left(\frac{1}{\sqrt{2}}, \frac{1}{\sqrt{2}}, 0,0, \ldots\right)$ and $y=(1,-1,0,0, \ldots)$. In this section we answer the question posed in [KMT] by showing that the inequalities obtained in that paper are indeed equalities. Then we use this computation to conclude that the space $\ell_{2, \infty}$ is a limiting space for both Theorem 1 and Theorem 2, i.e., that both theorems are sharp.

Theorem 4. $C_{\mathrm{NJ}}\left(\ell_{2, \infty}\right)=\frac{3}{2}$ and $J\left(\ell_{2, \infty}\right)=1+\frac{1}{\sqrt{2}}$.

Proof. We just need to prove that $C_{\mathrm{NJ}}\left(\ell_{2, \infty}\right) \leq \frac{3}{2}$ and $J\left(\ell_{2, \infty}\right) \leq 1+\frac{1}{\sqrt{2}}$. Consider any two vectors $x$ and $y$ in $\ell_{2, \infty}$, not simultaneously equal to zero, and let us see that

$$
c(x, y)=\frac{\|x+y\|_{2, \infty}^{2}+\|x-y\|_{2, \infty}^{2}}{2\left(\|x\|_{2, \infty}^{2}+\|y\|_{2, \infty}^{2}\right)} \leq \frac{3}{2},
$$


and also that if $\|x\|_{2, \infty} \leq 1$ and $\|y\|_{2, \infty} \leq 1$, then

$$
j(x, y)=\min \left\{\|x+y\|_{2, \infty},\|x-y\|_{2, \infty}\right\} \leq 1+\frac{1}{\sqrt{2}} .
$$

Our initial strategy will be to reduce the general situation to a more restrictive and easier to handle one.

Step 1. It suffices to prove that $c(x, y) \leq \frac{3}{2}$ and $j(x, y) \leq 1+\frac{1}{\sqrt{2}}$, for $x, y \in B_{l_{2, \infty}}$ satisfying the conditions (a.1), (a.2) and (a.3) below.

$$
\begin{array}{ll}
\text { (a.1) } & \|x+y\|_{2, \infty}=\left\|(x+y)^{+}\right\|_{2} . \\
\text { (a.2) }\|x-y\|_{2, \infty}=\left\|(x-y)^{+}\right\|_{2} . \\
\text { (a.3) } x=x^{+} .
\end{array}
$$

The reason why this can be done is based in the following claim. For any $u, v \in B_{l_{2, \infty}}$ there exist $x, y \in B_{l_{2, \infty}}$ such that the following conditions are satisfied:

(a) $\|x\|_{2, \infty} \leq\|u\|_{2, \infty},\|y\|_{2, \infty} \leq\|v\|_{2, \infty}$,

(b) $x, y$ satisfy the conditions (a.1), (a.2) and (a.3),

(c) $c(u, v) \leq c(x, y)$ and $j(u, v) \leq j(x, y)$.

Indeed, for $u, v \in B_{l_{2, \infty}}$, we may assume without loss of generality that $\|u+v\|_{2, \infty}=\left\|(u+v)^{+}\right\|_{2}$ and $\|u-v\|_{2, \infty}=\left\|(u-v)^{+}\right\|_{2}$. Now, put $x=u^{+}$ and $y=v$. Then we have that (a) and (b) hold. Moreover, (c) also holds by means of the following inequalities:

$$
\left\|(x+y)^{-}\right\|_{2} \leq\left\|(u+v)^{-}\right\|_{2} \leq\left\|(u+v)^{+}\right\|_{2} \leq\left\|(x+y)^{+}\right\|_{2}
$$

and

$$
\left\|(x-y)^{-}\right\|_{2} \leq\left\|(u-v)^{-}\right\|_{2} \leq\left\|(u-v)^{+}\right\|_{2} \leq\left\|(x-y)^{+}\right\|_{2} .
$$

Let us observe that $u(i) \leq x(i)$ for all positive integer $i$. To prove the third inequality in (a.3.1), it suffices to show that, by definition of the norm $\|\cdot\|_{2}$,

$$
(u+v)^{+}(i) \leq(x+y)^{+}(i)
$$

for each positive integer $i$ with $u(i)+v(i) \geq 0$. Indeed, for such $i$,

$$
0 \leq(u+v)^{+}(i)=u(i)+v(i) \leq x(i)+y(i)=(x+y)^{+}(i) .
$$

To prove the first inequality in (a.3.1), it suffices to observe that

$$
(x+y)^{-}(i) \leq(u+v)^{-}(i)
$$

for each positive integer $i$ with $x(i)+y(i) \leq 0$, since, for such $i$,

$$
0 \leq(x+y)^{-}(i)=-x(i)-y(i) \leq-u(i)-v(i)=(u+v)^{-}(i) .
$$

The second inequality of (a.3.1) is trivial, so the inequalities in (a.3.1) follow.

Similarly, we obtain (a.3.2).

Step 2. If $x$ and $y$ satisfy (a.1), (a.2) and (a.3), then

$$
\|x+y\|_{2, \infty}^{2}+\|x-y\|_{2, \infty}^{2} \leq 2\|x\|_{2}^{2}+2\|y\|_{2}^{2}-\left(\|P(y)\|_{2}-\|P(x)\|_{2}\right)^{2},
$$

where, for $u \in \ell_{2}, P(u) \in \ell_{2}$ is defined as

$$
P(u)(i)= \begin{cases}u(i), & \text { if }|y(i)| \geq|x(i)|, \\ 0, & \text { otherwise }\end{cases}
$$


To be convinced that the relation (1) is true, check the following inequalities using the definition of the norm $\|\cdot\|_{2}$, the parallelogram law, some arithmetic calculus and the Cauchy-Schwarz inequality:

$$
\begin{aligned}
\| x+ & y\left\|_{2, \infty}^{2}+\right\| x-y\left\|_{2, \infty}^{2}=\right\|(x+y)^{+}\left\|_{2}^{2}+\right\|(x-y)^{+} \|_{2}^{2} \\
& =\|x+y\|_{2}^{2}+\|x-y\|_{2}^{2}-\left\|(x+y)^{-}\right\|_{2}^{2}-\left\|(x-y)^{-}\right\|_{2}^{2} \\
& =2\|x\|_{2}^{2}+2\|y\|_{2}^{2}-\left\|(x+y)^{-}\right\|_{2}^{2}-\left\|(x-y)^{-}\right\|_{2}^{2} \\
& =2\|x\|_{2}^{2}+2\|y\|_{2}^{2}-\|P(x)\|_{2}^{2}-\|P(y)\|_{2}^{2}+2\left\langle P(x), P(y)^{+}+P(y)^{-}\right\rangle \\
& \leq 2\|x\|_{2}^{2}+2\|y\|_{2}^{2}-\|P(x)\|_{2}^{2}-\|P(y)\|_{2}^{2}+2\|P(x)\|_{2}\|P(y)\|_{2} \\
& =2\|x\|_{2}^{2}+2\|y\|_{2}^{2}-\left(\|P(y)\|_{2}-\|P(x)\|_{2}\right)^{2} .
\end{aligned}
$$

Step 3. Let us see now that $c(x, y) \leq \frac{3}{2}$. Of course, we assume that $x$ and $y$ satisfy (a.1), (a.2) and (a.3), and hence inequality (1).

Observe that

$$
\|y\|_{2}^{2}=\|y\|_{2, \infty}^{2}+m(y)^{2}
$$

where $m(y)=\min \left\{\left\|y^{+}\right\|_{2},\left\|y^{-}\right\|_{2}\right\}$, which combined with (1) gives that

$$
\begin{aligned}
c(x, y) & =\frac{\|x+y\|_{2, \infty}^{2}+\|x-y\|_{2, \infty}^{2}}{2\left(\|x\|_{2, \infty}^{2}+\|y\|_{2, \infty}^{2}\right)} \\
& \leq \frac{2\|x\|_{2}^{2}+2\|y\|_{2}^{2}-\left(\|P(y)\|_{2}-\|P(x)\|_{2}\right)^{2}}{2\left(\|x\|_{2}^{2}+\|y\|_{2, \infty}^{2}\right)} \\
& =1+\frac{2 m(y)^{2}-\left(\|P(y)\|_{2}-\|P(x)\|_{2}\right)^{2}}{2\left(\|x\|_{2}^{2}+\|y\|_{2, \infty}^{2}\right)} .
\end{aligned}
$$

Before we continue with the chain of inequalities (2), we shall control some terms appearing there. Observe that $\|y\|_{2}^{2} \geq 2 m(y)^{2}$ and that $\|y-P(y)\|_{2}^{2} \leq\|x-P(x)\|_{2}^{2}$. Thus

$$
\begin{aligned}
m(y)^{2} & \leq \frac{1}{2}\|y\|_{2}^{2} \\
& =\frac{1}{2}\left(\|y-P(y)\|_{2}^{2}+\|P(y)\|_{2}^{2}\right) \\
& \leq \frac{1}{2}\left(\|x-P(x)\|_{2}^{2}+\|P(y)\|_{2}^{2}\right) \\
& =\frac{1}{2}\|x\|_{2}^{2}+\frac{1}{2}\left(\|P(y)\|_{2}^{2}-\|P(x)\|_{2}^{2}\right) .
\end{aligned}
$$

Then, using the above inequality and the fact that $\|P(x)\|_{2} \leq\|x\|_{2}$,

$$
\begin{aligned}
& m(y)^{2}-\left(\|P(y)\|_{2}-\|P(x)\|_{2}\right)^{2} \\
& \leq \frac{1}{2}\|x\|_{2}^{2}+\frac{1}{2}\left(\|P(y)\|_{2}^{2}-\|P(x)\|_{2}^{2}\right)-\left(\|P(y)\|_{2}-\|P(x)\|_{2}\right)^{2} \\
& =\frac{1}{2}\|x\|_{2}^{2}-\frac{1}{2}\|P(y)\|_{2}^{2}+2\|P(y)\|_{2}\|P(x)\|_{2}-\frac{3}{2}\|P(x)\|_{2}^{2} \\
& \leq \frac{1}{2}\|x\|_{2}^{2}+\frac{1}{2}\|P(x)\|_{2}^{2} \\
& \leq\|x\|_{2}^{2} .
\end{aligned}
$$


With this last estimate, and having in mind that $m(y) \leq\|y\|_{2, \infty}$, we can continue with the inequalities (2) as follows:

$$
\begin{aligned}
c(x, y) & \leq 1+\frac{m(y)^{2}+\|x\|_{2}^{2}}{2\left(\|x\|_{2}^{2}+\|y\|_{2, \infty}^{2}\right)} \\
& \leq 1+\frac{\|y\|_{2, \infty}^{2}+\|x\|_{2}^{2}}{2\left(\|x\|_{2}^{2}+\|y\|_{2, \infty}^{2}\right)} \\
& =\frac{3}{2} .
\end{aligned}
$$

Step 4. Let us see that $j(x, y) \leq 1+\frac{1}{\sqrt{2}}$, provided that $x$ and $y$ are in the closed unit ball of $\ell_{2, \infty}$.

By Step 1, we may assume that $x$ and $y$ satisfy (a.1), (a.2) and (a.3). Then relation (1)-(3) holds. Also, since $\|y-P(y)\|_{2} \leq\|x-P(x)\|_{2}$ and $\|x\|_{2}=\|x\|_{2, \infty} \leq$ 1 , we deduce from (3) that

$$
\|y\|_{2}^{2} \leq 1+\left(\|P(y)\|_{2}^{2}-\|P(x)\|_{2}^{2}\right) .
$$

Thus, using this last estimate and (1), we obtain

$$
\begin{aligned}
2 j(x, y)^{2} & \leq\|x+y\|_{2, \infty}^{2}+\|x-y\|_{2, \infty}^{2} \\
& \leq 2\|x\|_{2}^{2}+2\|y\|_{2}^{2}-\left(\|P(y)\|_{2}-\|P(x)\|_{2}\right)^{2} \\
& \leq 4+2\left(\|P(y)\|_{2}^{2}-\|P(x)\|_{2}^{2}\right)-\left(\|P(y)\|_{2}-\|P(x)\|_{2}\right)^{2} .
\end{aligned}
$$

Hence, we have obtained a bound of the form

$$
2 j(x, y)^{2} \leq 4+2 a-\left(\sqrt{\|P(x)\|_{2}^{2}+a}-\|P(x)\|_{2}\right)^{2},
$$

where $a=\|P(y)\|_{2}^{2}-\|P(x)\|_{2}^{2} \geq 0$, and $0 \leq\|P(x)\|_{2} \leq 1$.

We can continue our argument as follows: since $a \geq 0$ and $\|P(x)\|_{2} \leq 1$, an elementary calculation shows that

$$
\sqrt{\|P(x)\|_{2}^{2}+a}-\|P(x)\|_{2} \geq \sqrt{1+a}-1,
$$

and then

$$
\begin{aligned}
2 j(x, y)^{2} & \leq 4+2 a-(\sqrt{1+a}-1)^{2} \\
& =2+a+2 \sqrt{1+a} .
\end{aligned}
$$

This last inequality solves our problem for $a \leq 1: 2 j(x, y)^{2} \leq 3+2 \sqrt{2}$ and then $j(x, y) \leq 1+\frac{1}{\sqrt{2}}$. But it does not work for $a>1$. In this final case, since $\|x\|_{2} \leq 1$ and $\|y\|_{2} \leq \sqrt{2}$,

$$
\begin{aligned}
1 & \leq a=\|P(y)\|_{2}^{2}-\|P(x)\|_{2}^{2} \\
& =\left(\|P(y)\|_{2}-\|P(x)\|_{2}\right)\left(\|P(y)\|_{2}+\|P(x)\|_{2}\right) \\
& \leq\left(\|P(y)\|_{2}-\|P(x)\|_{2}\right)(\sqrt{2}+1),
\end{aligned}
$$

and hence, using (1),

$$
2 j(x, y)^{2} \leq 6-\left(\frac{1}{\sqrt{2}+1}\right)^{2}=3+2 \sqrt{2},
$$

from which $j(x, y) \leq 1+\frac{1}{\sqrt{2}}$. 
Remark 5. It is easy to see that $\mu\left(\ell_{2, \infty}\right)=\sqrt{2}$, and since $\ell_{2, \infty}$ lacks normal structure [B] we conclude from Theorem 4 that the results obtained in Theorem 1 and Theorem 2 are sharp.

\section{REFERENCES}

[B] W.L. Bynum, Normal structure coefficients for Banach spaces. Pacific J. Math. 86 (1980), 427-436. MR0590555 (81m:46030)

[DPS] S. Dhompongsa, P. Piraisangunn and S. Saejung, Generalised Jordan-von Neumann constants and uniform normal structure. Bull. Austral. Math. Soc. 67-2 (2003), 225-240. MR $1972712(2005 \mathrm{c}: 46015)$

[DKT] S. Dhompongsa, A. Kaewkhao and S. Tasena, On a generalized James constant. J. Math. Anal. Appl. 285 (2003), 419-435. MR2005130 (2004f:46020)

[GL1] J. GaO AND K.S. LAU, On the geometry of spheres in normed linear spaces. J. Austral. Math. Soc. (Series A) 48 (1990), 101-112. MR1026841 (91e:46025)

[GL2] J. GaO AND K.S. LAU, On two classes of Banach spaces with uniform normal structure. Studia Math. 99 (1991), 41-56. MR.1120738 (92h:46017)

[G-K] K. Goebel and W.A. Kirk, Topics in metric fixed point theory. Cambridge Univ. Press 1990. MR 1074005 (92c:47070)

[JL] A. JimÉnEZ-MElado AND E.Llorens-Fuster, The fixed point property for some uniformly nonsquare Banach spaces. Boll. Un. Mat. Ital. (7) 10-A (1996), 587-595. MR:1420941 (97m:47080)

[KMT] M. Kato, L. MaligRanda And Y. TAKahashi, On James and Jordan-von Neumann constants and the normal structure coefficient of Banach spaces. Studia Math. 144 (2001), no. 3, 275-295. MR1829721(2002k:46035)

[KT] M. Kato AND Y. TAKAHASHI, On the von Neumann-Jordan constant for Banach spaces. Proc. A. M. S. 125 (4) (1997), 1055-1062. MR1371131 (97f:46017)

[Kh Ki] M. A. Khamsi And W.A.KIRK, An introduction to metric spaces and fixed point theory. Pure and Appl. Math., Wiley and Sons, N.Y. 2001. MR.1818603 (2002b:46002)

[Ki] W.A. KIRK, A fixed point theorem for mappings which do not increase distances. Amer. Math. Monthly 72 (1965), 1004-1006. MR0189009 (32:6436)

[Ki S] W.A.KIRK AND B. Sims Eds., Handbook of metric fixed point theory. Kluwer, Dordrecht, 2001. MR.1904271 (2003b:47002)

[P] S. Prus, Geometrical background of Metric Fixed Point Theory, in Handbook of Metric Fixed Point Theory., W. A. Kirk and B. Sims eds., Kluwer Academic Publ. 2001, 93-132. MR.1904275 (2003m:46020)

[S] B. Sims A class of spaces with weak normal structure. Bull. Austral. Math. Soc. 50 (1994), 523-528. MR.1274532 (95c:46025)

Departamento de Análisis Matemático, Universidad de Málaga, Facultad de Ciencias, 29071 Málaga, Spain

E-mail address: melado@uma.es

Departamento de Análisis Matemático, Facultad de Matemáticas, 46100 Burjassot, Valencia, Spain

E-mail address: enrique.1lorens@uv.es

Department of Mathematics, Khon Kaen University, Khon Kaen, 40002, Thailand

E-mail address: satitz@yahoo.com 